\title{
Laboratory test result interpretation for primary care doctors in South Africa
}

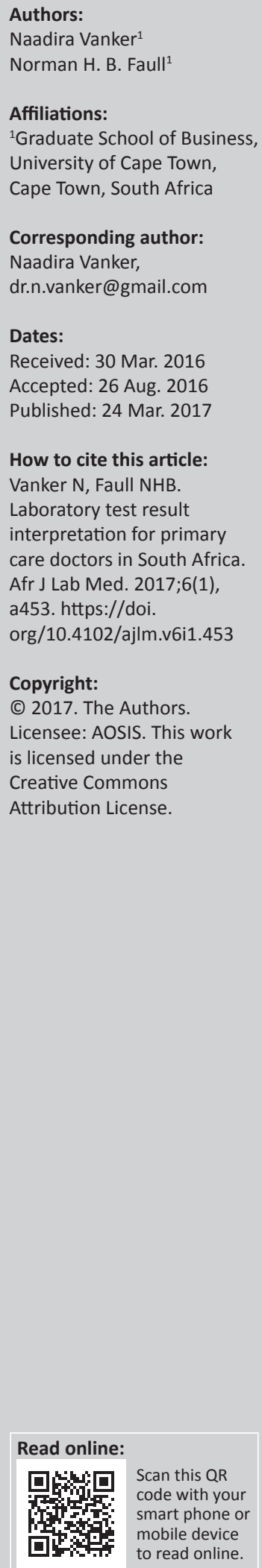

Background: Challenges and uncertainties with test result interpretation can lead to diagnostic errors. Primary care doctors are at a higher risk than specialists of making these errors, due to the range in complexity and severity of conditions that they encounter.

Objectives: This study aimed to investigate the challenges that primary care doctors face with test result interpretation, and to identify potential countermeasures to address these.

Methods: A survey was sent out to 7800 primary care doctors in South Africa. Questionnaire themes included doctors' uncertainty with interpreting test results, mechanisms used to overcome this uncertainty, challenges with appropriate result interpretation, and perceived solutions for interpreting results.

Results: Of the 552 responses received, the prevalence of challenges with result interpretation was estimated in an average of $17 \%$ of diagnostic encounters. The most commonly-reported challenges were not receiving test results in a timely manner ( $51 \%$ of respondents) and previous results not being easily available (37\%). When faced with diagnostic uncertainty, $84 \%$ of respondents would either follow-up and reassess the patient or discuss the case with a specialist, and $67 \%$ would contact a laboratory professional. The most useful test utilisation enablers were found to be: interpretive comments (78\% of respondents), published guidelines (74\%), and a dedicated laboratory phone line (72\%).

Conclusion: Primary care doctors acknowledge uncertainty with test result interpretation. Potential countermeasures include the addition of patient-specific interpretive comments, the availability of guidelines or algorithms, and a dedicated laboratory phone line. The benefit of enhanced test result interpretation would reduce diagnostic error rates.

\section{Introduction}

Laboratory services play an integral role in the healthcare system - from primary- through to tertiary-level care - since diagnostic tests can either confirm or exclude a tentative diagnosis, or screen for potential diseases. ${ }^{1}$ The underlying purpose of laboratory testing lies in the association between laboratory test results and the potential to improve a patient's health status. This requires ordering a test when appropriate and necessary, accurate interpretation, and acting upon the result. $^{2}$

Primary care doctors are usually the point of entry into a healthcare system, and are consequently exposed to a variety of medical conditions that range in both complexity and severity. It is postulated that these doctors are therefore at a higher risk of making medical errors than specialists. ${ }^{3}$ Due to the variety and intricacies of laboratory tests available, there is the potential for test-related errors to occur in a range of clinical conditions, that may result in significant patient harm. ${ }^{4}$ Studies have shown that between $15 \%$ and $54 \%$ of errors occurring at a primary healthcare level are related to the testing process. ${ }^{5}$ Diagnostic errors can be due to three underlying causes, namely: no identifiable fault, system-related, and cognitive. Cognitive errors are caused by incorrect interpretation of available information and may be caused by faulty knowledge, faulty data gathering, or faulty synthesis of data. A large-scale study found that up to $74 \%$ of diagnostic errors are either completely or in part due to cognitive failures. ${ }^{6}$ This suggests that many diagnostic errors are related to misunderstanding or misinterpreting the available information.

Studies have shown that primary care doctors face uncertainty when interpreting clinical laboratory reports. ${ }^{7,9,10}$ A nationwide study involving 1768 primary care physicians was conducted in the United States to determine the challenges that this group faces with laboratory test ordering and 
result interpretation. The findings were that clinicians experienced uncertainty due to inconsistencies in the receipt of results, problems with the report format, and difficulties with interpretation of results in $8.3 \%$ of diagnostic encounters. ${ }^{7}$ Research conducted in South Africa also demonstrated the uncertainty that doctors experience with laboratory test result interpretation. This study investigated how confident interns were with requesting biochemical tests and interpreting the results. The study found that although these junior doctors were fairly confident when dealing with common investigations, they experienced challenges with interpreting the results of more complex and less common tests. Of the 61 respondents, $23 \%$ reported a lack of confidence in interpreting the results of complex tests. ${ }^{8}$

This explorative study investigated the problems and challenges that primary healthcare doctors in South Africa face with the interpretation of clinical laboratory test results. A secondary aim of the study was to identify potential countermeasures to address these challenges.

\section{Methods}

\section{Ethical considerations}

Ethical approval was granted by the Commerce Faculty Ethics in Research Committee, Graduate School of Business, University of Cape Town. Anonymity was maintained throughout the process. Informed consent was obtained through a cover letter containing the survey link. The surveys were completed online, with all responses anonymised through the system. The researchers did not have access to respondents' identifying or personal information.

\section{Study design}

This research was based on a study conducted by Hickner et al., entitled 'Primary care physicians' challenges in ordering clinical laboratory tests and interpreting results'.? The original survey was developed through an inductive approach, using information obtained from three focus groups comprising 27 primary care doctors, as well as from a panel of experts working in primary healthcare and laboratory medicine. The questionnaire was authorised for use by the Office of the Associate Director for Science at the US Centers for Disease Control and Prevention.

The original nineteen-part questionnaire was reduced to nine sections to focus on the challenges that primary healthcare doctors face with clinical laboratory result interpretation. The survey themes included doctors' uncertainty with interpreting test results, mechanisms they use to overcome this uncertainty, challenges with appropriate result interpretation, and perceived solutions to interpreting test results. The questionnaire categories were as follows: (1) demographic information; (2) information about the doctor's practice; (3) interpretation uncertainty; (4) the diagnostic evaluation process; (5) laboratory consultation; and (6) test utilisation enablers. Questions related to the doctor's practice included: whether the doctor was a general practitioner or specialist; the number of years in practice; the predominant categories of tests ordered (i.e. diagnostic tests, chronic disease monitoring, or routine screening); the number of patients seen per week; the number of tests ordered per week; and the number of tests per week that were associated with interpretation uncertainty. Two questions were added to the demographic section of the questionnaire to determine whether the South African doctor worked in a rural, semiurban or urban practice and whether he/she predominantly made use of private pathology laboratories or the parastatal (National Health Laboratory Service) laboratory. However, it was not ascertained whether the majority of patients seen were hospitalised or out-patients. Responses were predominantly chosen from a list of five-point graded options, but there was space given for open-ended responses. Response options ranged from: 'extremely useful' to 'not at all useful', 'extremely important' to 'not at all important', 'extremely well' to 'not at all well', and 'extremely problematic' to 'not at all problematic'.

\section{Survey administration}

For this cross-sectional study, questionnaires were sent out electronically using a survey link to the approximately 7800 primary care doctors in the South African Medical Association database. The survey was sent on 13 October 2015 and remained open for responses until 13 November 2015.

\section{Analysis}

Response data from the surveys were exported to Microsoft Excel (Microsoft Corp., Redmond, Washington, United States) and analysed using the IBM SPSS Statistics ${ }^{\circledR}$ package (IBM SPSS Statistics for Macintosh, Version 22.0.; IBM Corp., Armonk, New York, United States). The qualitative responses were analysed quantitatively using descriptive statistics to determine relative frequencies. Results presented were based on the number of respondents who selected the top two responses from the five-point scale - namely, 'extremely and very useful', 'extremely and very important', 'extremely and very well', or 'extremely and very problematic'. Open-ended responses received were reported as 'other' in the figures below.

\section{Results \\ Overview and respondent characteristics}

Of the approximately 7800 questionnaires sent out, 552 completed questionnaires were received, equating to a response rate of $7 \%$. Incomplete questionnaires were excluded from the analysis, so as not to skew the results. Although the survey was sent to doctors registered in the South African Medical Association database as general and/ or independent practitioners, this database included a few doctors who were either in training or were qualified specialists. Table 1 describes the doctors' practice characteristics and test utilisation information. Of note, respondents saw an average of 115 patients per week, ordered an average of 24 tests per week, and experienced uncertainty 
TABLE 1: Respondents' practice characteristics and laboratory test utilisation information, South Africa, 13 October 2015-13 November 2015.

\begin{tabular}{|c|c|c|c|}
\hline Variables & Number of respondents & Result & Range \\
\hline Average number of years in practice & 552 & 13 years & $1-47$ years \\
\hline \multicolumn{4}{|l|}{ General or specialist practitioner } \\
\hline General Practitioner & 513 & $93 \%$ & - \\
\hline Specialist $^{\dagger}$ & 39 & $7 \%$ & - \\
\hline \multicolumn{4}{|l|}{ Location of practice } \\
\hline Urban & 298 & $54 \%$ & - \\
\hline Peri-urban & 133 & $24 \%$ & - \\
\hline Rural & 121 & $22 \%$ & - \\
\hline \multicolumn{4}{|l|}{ Type of laboratory utilised most frequently } \\
\hline $\begin{array}{l}\text { Government/Parastatal (National Health Laboratory } \\
\text { Service) }\end{array}$ & 304 & $55 \%$ & - \\
\hline Private & 248 & $45 \%$ & - \\
\hline \multicolumn{4}{|l|}{$\begin{array}{l}\text { Predominant categories } \\
\text { ( } 50-100 \% \text { of tests) ordered by individual doctors }\end{array}$} \\
\hline Diagnostic tests & 248 & $45 \%$ & - \\
\hline Chronic disease monitoring & 138 & $25 \%$ & - \\
\hline Routine screening & 94 & $17 \%$ & - \\
\hline No predominance & 72 & $13 \%$ & - \\
\hline Average number of patients seen per week & 552 & 115 patients & $3-500$ patients \\
\hline Average number of laboratory tests ordered per week & 552 & 24 tests & $0-200$ tests \\
\hline $\begin{array}{l}\text { Average number of tests per week for which there is } \\
\text { uncertainty in result interpretation }\end{array}$ & 552 & 4 tests & $0-30$ tests \\
\hline
\end{tabular}

${ }^{\dagger}$, Fields of specialty included: family medicine, internal medicine, emergency medicine, palliative medicine, HIV care, sports medicine, psychiatry, anaesthetics, paediatrics, public health, general surgery, and neurosurgery.

in result interpretation for four of these tests. This equates to challenges in the interpretation of approximately $17 \%$ of test results.

\section{Challenges with laboratory test results}

The challenges that doctors experienced with laboratory test results (Figure 1) were selected from a list of possible options and rated using the five-point scale. The most prominent problems were related to accessing results with $51 \%$ of respondents reporting that not receiving results in a timely manner was very problematic and 37\% reporting problems with availability of previous test results. (The timeliness of the receipt of results was as perceived by the respondents and was not quantified or defined in the question.) The nexthighest reported type of challenge related to the result being incompatible with the patient's clinical picture, which could either be seen as an inconsistent result (27\%) or a laboratory error $(28 \%)$.

Although not strictly related to challenges with laboratory test results, a few respondents did report challenges with laboratory access and financing in the open-ended part of this section. These challenges included: difficulties for rural hospitals or practices to get samples to a laboratory, the unavailability of specialised tests (e.g., B-type natriuretic peptide and Helicobacter pylori $\mathrm{IgG}$ ), and medical aids not authorising or paying for tests.

\section{Diagnostic evaluation process}

The majority of doctors (66\%) typically used a core set of 20 or fewer clinical laboratory diagnostic tests. When faced with diagnostic uncertainty, most respondents (67\%) reported always double-checking with another doctor or electronic resources (e.g., UpToDate, WebMD, patient.co.uk, etc.) if they

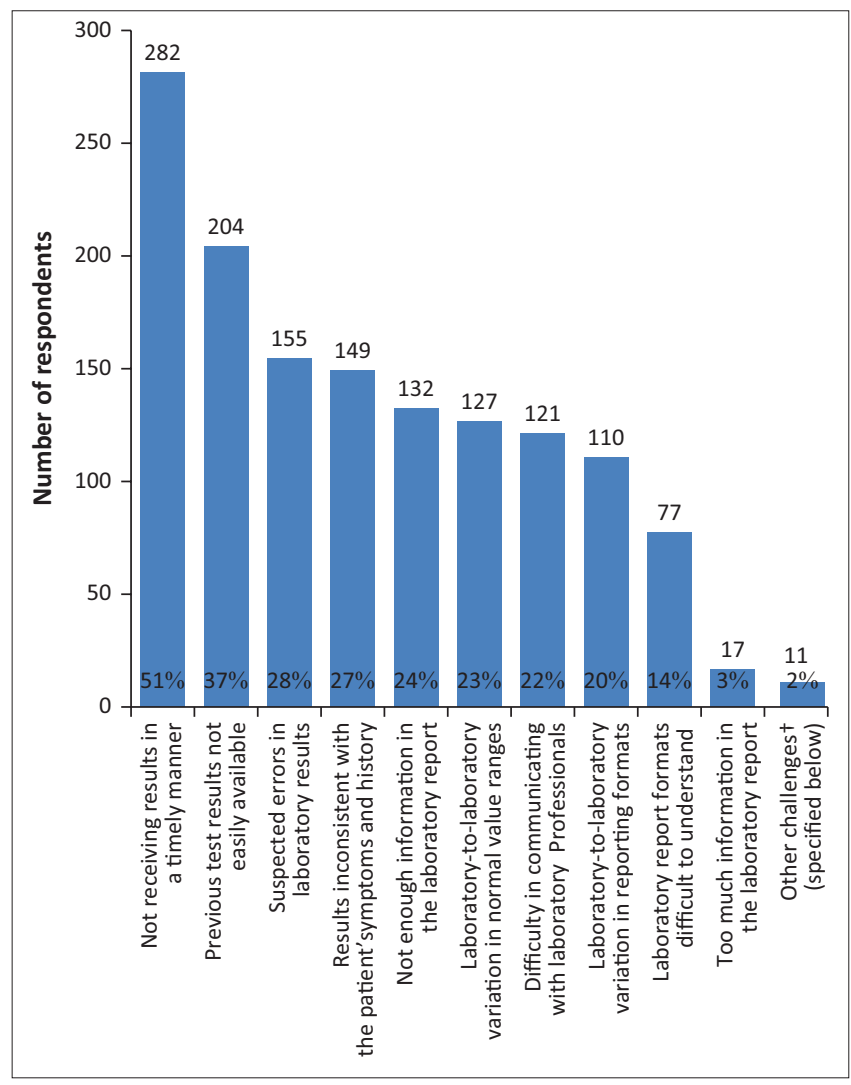

$\dagger$, Other challenges included: problems with determining the significance of abnormal results (e.g. macrocytosis) and laboratory test results getting lost.

FIGURE 1: Challenges that doctors face when using laboratory test results, South Africa, 13 October 2015-13 November 2015.

doubted their decision. Even when confident in their pre-test diagnoses, $42 \%$ of doctors would still think 'what else could it be?'. While $58 \%$ of clinicians were concerned about overtesting their patients, only $33 \%$ were concerned about undertesting patients. 


\section{Interpretation uncertainty}

When faced with diagnostic uncertainty in a difficult or unusual case (Figure 2), most respondents would either follow-up and reassess the patient $(84 \%)$ or review the patient's history and physical findings (82\%). Eighty-four percent of primary care doctors also found it very useful to discuss the case with a specialist.

\section{Laboratory consultation}

In a variety of contexts, most respondents found communication with the laboratory to be useful (Figure 3). Of note, $82 \%$ of doctors found it very useful for the laboratory to contact the clinician with critically abnormal results.

In general, only about one-third of respondents noted very important reasons as to why they did not frequently contact laboratory professionals. These reasons included: difficulties in contacting the person who could answer their questions $(36 \%)$; not knowing whom to contact $(33 \%)$; difficulties in getting through to the laboratory (32\%); and not having a working relationship with laboratory professionals $(27 \%)$. Only $11 \%$ reported that they did not contact the laboratory because they felt that they had received unreliable information during previous interactions. A small number (2\%) of individuals reported specific problems with public sector laboratories wherein they felt that laboratory staff were unhelpful regarding lost or rejected specimens, and inaccurate, delayed or urgent results. A lack of access to pathologists at certain regional laboratories was also noted as a problem.

\section{Test utilisation enablers}

Test utilisation enablers (Figure 4) are methods that have been developed to assist clinicians in using diagnostic laboratory

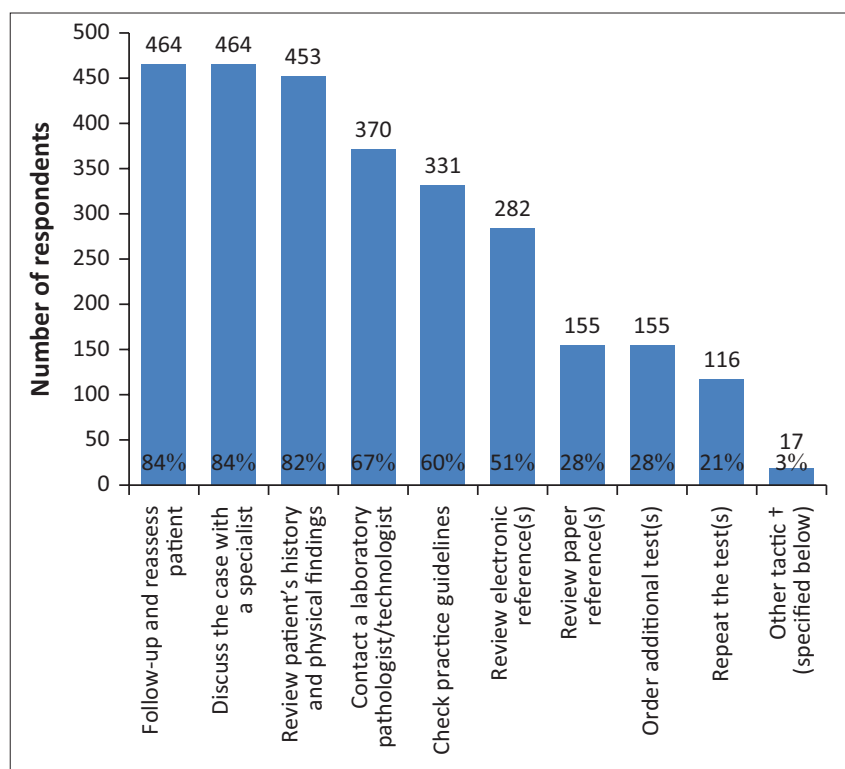

†, Other tactics included: ignoring inconsistent results, referring the patient from a primary care level practice to hospital or a specialist, and discussing the case with non-specialist colleagues.

FIGURE 2: Tactics employed by doctors to deal with test interpretation uncertainty, South Africa, 13 October 2015-13 November 2015.

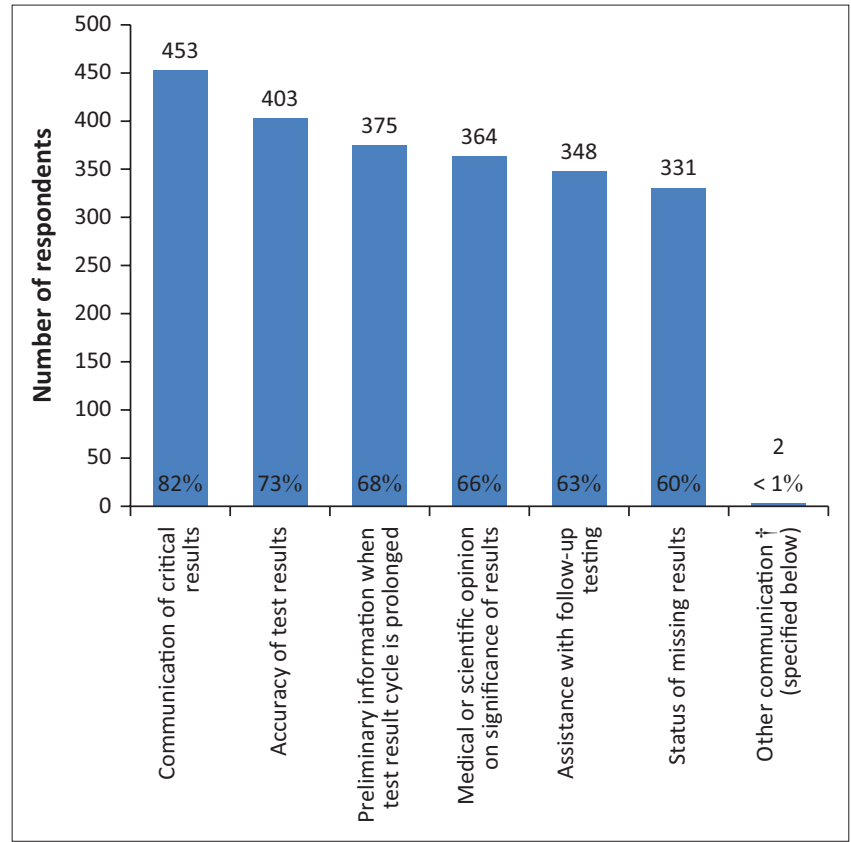

†, Other communication included: a WhatsApp ${ }^{\circledR}$ group for the microbiology laboratory where doctors receive immediate notification when blood culture or cerebrospinal fluid results are available, which aids in reducing time to appropriate treatment.

FIGURE 3: Usefulness of laboratory communication/consultation, South Africa, 13 October 2015-13 November 2015.

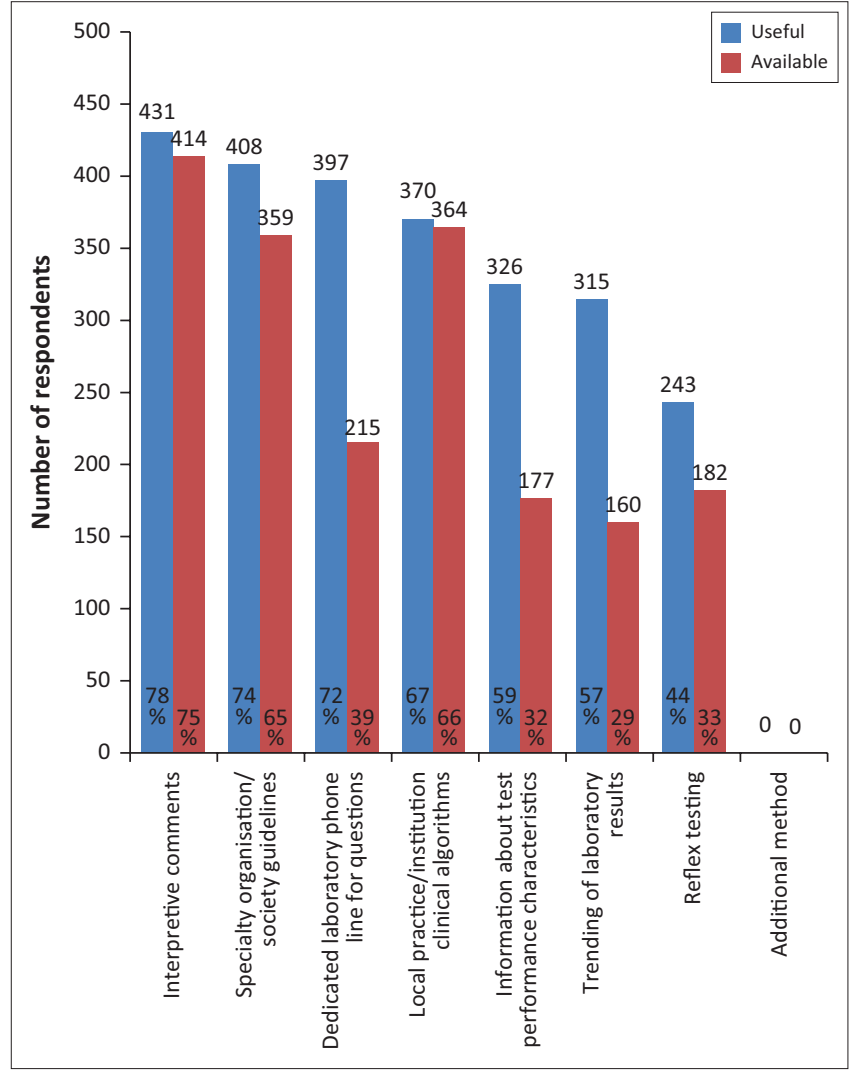

FIGURE 4: Usefulness and availability of test utilisation enablers, South Africa, 13 October 2015-13 November 2015.

testing more effectively. Interpretive comments - comments provided with the test result to give additional information on the meaning of the results - were reported by $78 \%$ of respondents as very useful. Seventy-four percent of doctors found guidelines - aids published by specialty organisations 
or societies for the interpretation of patient's test results based on clinical presentation usually guided by decision trees - to be useful. Similarly, clinical algorithms - guidelines used within local practices or institutions - were reported as very useful by $67 \%$ of doctors. Seventy-two percent of the respondents reported that a dedicated laboratory phone was useful; however, only $39 \%$ of respondents had access to a dedicated laboratory phone line. Information on test performance characteristics, such as sensitivity, specificity, and likelihood ratios, were also reported as very useful by $59 \%$ of respondents, but were only available to $32 \%$. The test utilisation enablers considered useful were selected from the options presented in the five-point scale questions, and no additional enablers were suggested by respondents in the open-ended response section.

When compared to urban and peri-urban respondents, rural doctors experienced considerably lower availability of interpretive comments, information on test performance characteristics, trending of laboratory results (when previous results are compared with current results), and reflex testing (a test performed by the laboratory in response to results from a previous test) (Table 2). In contrast, the rural doctors reported increased availability of a dedicated laboratory phone line.

\section{Further comments}

Open-ended feedback on result interpretation was also elicited from respondents. Resultsincluded acknowledgement that the 'interpretation of results are [sic] critical to reliably apply the blood results to our patients in terms of diagnosis, screening and monitoring their pathology'. A number of respondents felt that the interpretive comments currently received were not specific to patients' age, sex, clinical picture or previous results, but were instead based on general information. Furthermore, these comments did not include recommendations for further testing or treatment. Specific challenges were noted in the interpretation of microbiology, serology (especially hepatitis B), and discordant HIV results. In contrast to the challenges noted, certain private laboratories were identified and commended for the inclusion (where necessary) of interpretive comments written by pathologists.

\section{Discussion}

This study found that primary care doctors in South Africa experience challenges with laboratory test result interpretation in approximately $17 \%$ of their diagnostic encounters. By comparison, a similar study conducted in the United States found that primary care physicians reported uncertainty in $8.3 \%$ of diagnostic encounters. This emphasises the need for improved mechanisms and countermeasures to aid South African doctors with result interpretation.

The most common general challenges with laboratory test results reported by primary care doctors are related to receiving and accessing results - namely, not receiving results timeously and previous results not being easily available. Literature shows that over $80 \%$ of laboratories receive complaints about turn-around times, ${ }^{11}$ yet there are no universal evidence-based goals for laboratory processing times, and clinicians' expectations have often been found to be unreasonable. Nevertheless, laboratories should acknowledge customer dissatisfaction, and aim to provide results within a timeframe that is achievable by the laboratory and optimal for patient care. ${ }^{11}$ Trending of results is the displaying of a patient's previous results alongside the current test result to identify patterns of change and to enhance result interpretation. Over half of all respondents in our study found this to be an extremely or very useful test utilisation enabler, but less than a third reported its availability. Result trending has been shown to decrease time spent by clinicians on a case. ${ }^{12}$ Laboratories can play a role in addressing these challenges by determining appropriate turn-around times and communicating these times to the doctors, as well as by including previous test results on current reports to enable result trending. However, the availability of previous results requires integrated health information technology systems, which are not available in all healthcare environments. ${ }^{12}$

Although this study focused on the post-analytical phase of laboratory testing, the survey raised two questions around the analytic testing process and whether the clinicians experienced challenges with 'suspected errors in laboratory results' and 'results inconsistent with the patient's symptoms and history'. Inconsistent results were reported as a challenge by $27 \%$ of respondents and possible laboratory errors by $28 \%$. However, this did not include inquiry into errors occurring in the pre-analytical phase of testing, such as the mislabeling of samples. It has been found that pre-analytical errors account for $55 \%$ of laboratory errors causing a missed or delayed diagnosis. ${ }^{13}$ Therefore, clinicians should be aware that suspected errors or inconsistent results might be due to failures that occur outside the control of the laboratory.

TABLE 2: Availability of test utilisation enablers for the urban, peri-urban and rural doctor cohorts, $\uparrow$ South Africa, 13 October 2015-13 November 2015.

\begin{tabular}{|c|c|c|c|c|c|c|c|c|}
\hline \multirow[t]{2}{*}{ Test Utilisation Enablers } & \multicolumn{2}{|c|}{ Average \pm Standard Deviation } & \multicolumn{2}{|c|}{ Urban Doctors } & \multicolumn{2}{|c|}{ Peri-urban Doctors } & \multicolumn{2}{|c|}{ Rural Doctors } \\
\hline & $N$ & $\%$ & $N$ & $\%$ & $N$ & $(\%)$ & $N$ & $(\%)$ \\
\hline Interpretive comments & $414 \pm 39$ & $75 \pm 7$ & 238 & 80 & 102 & 77 & 79 & 65 \\
\hline Specialty organisation/society guidelines & $359 \pm 11$ & $65 \pm 2$ & 194 & 65 & 84 & 63 & 81 & 67 \\
\hline Dedicated laboratory phone line for questions & $215 \pm 50$ & $39 \pm 9$ & 110 & 37 & 49 & 37 & 63 & 52 \\
\hline Local practice/institution clinical algorithms & $364 \pm 39$ & $66 \pm 7$ & 200 & 67 & 97 & 73 & 71 & 59 \\
\hline Information on test performance characteristics & $177 \pm 33$ & $32 \pm 6$ & 98 & 33 & 49 & 37 & 31 & 26 \\
\hline Reflex testing & $182 \pm 28$ & $33 \pm 5$ & 107 & 36 & 44 & 33 & 32 & 26 \\
\hline
\end{tabular}

, The cohorts consisted of $298(54 \%)$ urban, $133(24 \%)$ peri-urban and $121(22 \%)$ rural medical practices. 
Our study found that primary care doctors find consultation with other clinicians or laboratory professionals to be an important mechanism in aiding test result interpretation. The majority of respondents reported a dedicated laboratory phone line to be an important test utilisation enabler, and, although this was only available to less than $40 \%$ of the total study population, over half of the rural doctor cohort had access to this service. This suggests that laboratories based in rural areas are trying to leverage their limited resources. A review of literature found that failures in communication between clinicians and the laboratory could negatively impact patient safety. ${ }^{5}$ Improving communication channels between the laboratory and clinical practitioners could lead to improved patient care and reduce unnecessary specialist referrals, which are at times requested purely for test result interpretation. ${ }^{7}$

The majority of survey respondents reported interpretive comments to be the most useful test utilisation enabler. Interpretive comments are added to a laboratory report in order to provide further information on the result and to aid in the diagnostic process. These comments can be provided by a qualified pathologist through technology-based interpretive algorithms and expert systems or through the addition of a 'canned' comment. A 'canned' comment is prewritten text that is added onto all results for a specific test, regardless of the actual result or the patient's clinical history, and is considered to be the least useful form of interpretive commenting. ${ }^{14}$ Respondents in our study supported this view when noting that interpretive comments that were not specific to the patient's age, sex, clinical picture or previous results, were not particularly useful. A study assessing the impact of narrative interpretations for complex laboratory tests found that the comments reduced the time taken and the number of tests required to reach a diagnosis and had an impact on the differential diagnosis. Furthermore, most respondents in that study felt that the interpretive comments helped prevent a misdiagnosis. ${ }^{15}$ The provision of high quality, patient-specific interpretive comments should improve patient care, decrease diagnostic errors, reduce costs, and enhance appropriate specialist referrals. ${ }^{16}$

The majority of respondents also reported guidelines or algorithms to be useful test-utilisation enablers. Clinical algorithms and practice guidelines are developed to provide a standardised, evidence-based approach to clinical processes in order to reduce error rates, improve clinical effectiveness, and enhance the quality of patient care. ${ }^{17}$ Clinical algorithms are particularly helpful in the interpretation of results for conditions that require a complex panel of tests for diagnosis, management, and monitoring of disease progression (e.g., diabetes mellitus). ${ }^{18}$ However, the availability of guidelines does not always ensure their use or result in changes in medical practices and behaviours. It is recommended that guidelines be disseminated through systems or accompanied by tools to facilitate their use and effectiveness. ${ }^{19}$ A study that compared the use of a technologybased expert system with conventional (non-computer-based) guidelines, found that the computer-based guideline system shortened the time taken to reach a diagnosis from (on average) 3.2 days to one day. ${ }^{20}$ The availability of guidelines or algorithms would be a useful countermeasure to aid doctors in interpreting complex tests and recommending further investigations that would guide management decisions. These guidelines may be integrated into the existing health information technology system, which may not be developed in certain settings, or can be in the form of applications that are uploaded onto independent mobile devices.

It has been found that clinicians are often unaware of whether their diagnoses at the time when they are making them are correct or erroneous. ${ }^{21}$ Therefore, interventions to reduce errors, such as medical decision support systems, should be embedded in a system rather than being made available only when perceived to be needed..$^{21}$ To standardise quality and improve efficiency, particularly in areas where human capital is limited, information technology can be leveraged. Studies have shown that health information technology can enhance delivery of care, reduce errors, and decrease utilisation of potentially inappropriate care. . $^{12,19,20}$ Twenty-two per cent of respondents work in rural areas and the availability of test utilisation enablers (including interpretive comments) in these areas is lower compared to urban and peri-urban areas. Furthermore, it was reported that certain regional laboratories lack access to a pathologist. In these cases, embedded technology-based solutions (such as expert systems for interpretive comments or integrated guidelines) may be particularly useful in assisting primary care doctors with test result interpretation.

\section{Limitations}

Response rates to surveys are reported to be $10 \%-20 \%,{ }^{22}$ but the response rate in our study was $7 \%$. This could be because the electronic platform used for the survey administration dissuaded doctors uncomfortable with the technology from participating; additionally, the length of time required to complete the survey (15 minutes) may have been considered too long. ${ }^{23}$ However, the study by Hickner et al., ${ }^{7}$ on which our study is based, had a response rate of $5.6 \%$, suggesting that this type of research may be associated with low response rates. A possible further limitation in this study is that the challenges faced by clinicians with result interpretation may have been under-reported. Research has shown that medical doctors have a tendency to display overconfidence, which can impact self-reported findings. ${ }^{24}$

\section{Conclusion}

Primary care doctors in South Africa acknowledge that they experience uncertainty when interpreting certain clinical laboratory test results. The most useful countermeasures and mechanisms identified by the doctors to improve this included: the addition of patient-specific interpretive comments; the availability of national or international guidelines or local clinical algorithms; and enhanced communication with the laboratory through a dedicated phone line. The ultimate benefit of enhanced test result interpretation would be reduced 
diagnostic error rates and a more efficient and effective primary healthcare system, which would reduce the rates of referral for secondary and tertiary levels of care.

\section{Acknowledgements}

We would like to thank all the respondents who took the time to complete this survey. We also wish to thank Ms Marilyn Myburgh from the South African Medical Association for her assistance with distributing the survey and Dr Julie Taylor of the US Centers for Disease Control and Prevention for allowing us use of the questionnaire.

\section{Competing interests}

The authors declare that they have no financial or personal relationships which may have inappropriately influenced them in writing this article.

\section{Sources of support}

None.

\section{Authors' contributions}

N.V. was the project leader who co-designed the project, collected and analysed the data, and contributed to writing and preparing the manuscript. N.H.B.F. was the academic supervisor who co-designed the project and contributed to preparing the manuscript.

\section{References}

1. Forsman RW. Why is the laboratory an afterthought for managed care organizations? Clin Chem. 1996;42(5):813-816.

2. Jackson BR. Managing laboratory test use: principles and tools. Clin Lab Med. 2007;27(4):733-748. https://doi.org/10.1016/j.cll.2007.07.009

3. Singh H, Giardina TD, Meyer AN, et al. Types and origins of diagnostic errors in primary care settings. JAMA Intern Med. 2013;173(6):418-425. https://doi. org/10.1001/jamainternmed.2013.2777

4. Clayton PD, Evans SR, Pryor T, et al. Bringing HELP to the clinical laboratory - use of an expert system to provide automatic interpretation of laboratory data. Ann Clin Biochem. 1987;24(Suppl 1):5-11.
5. Smith ML, Raab SS, Fernald DH, et al. Evaluating the connections between primary care practice and clinical laboratory testing: a review of the literature and call for laboratory involvement in the solutions. Arch Pathol Lab Med. 2013;137(1):120125. https://doi.org/10.5858/arpa.2011-0555-RA

6. Graber ML, Franklin N, Gordon R. Diagnostic error in internal medicine. Arch Intern Med. 2005;165(13):1493-1499. https://doi.org/10.1001/archinte.165.13.1493

7. Hickner J, Thompson PJ, Wilkinson T, et al. Primary care physicians' challenges in ordering clinical laboratory tests and interpreting results. J Am Board Fam Med. 2014;27(2):268-274. https://doi.org/10.3122/jabfm.2014.02.130104

8. Stanfliet JC, Macauley J, Pillay TS. Quality of teaching in chemical pathology: ability of interns to order and interpret laboratory tests. J Clin Pathol. 2009;62(7):664-666. https://doi.org/10.1136/jcp.2009.064824

9. Khromova V, Gray TA. Learning needs in clinical biochemistry for doctors in foundation years. Ann Clin Biochem. 2008;45(1):33-38. https://doi.org/10.1258/ acb.2007.007122

10. Gandhi TK, Kachalia A, Thomas EJ, et al. Missed and delayed diagnoses in the ambulatory setting: a study of closed malpractice claims. Ann Intern Med. 2006;145(7):488-496. https://doi.org/10.7326/0003-4819-145-7-200610030-00006

11. Hawkins RC. Laboratory turnaround time. Clin Biochem Rev. 2007;28(4):179.

12. Chaudhry B, Wang J, Wu S, et al. Systematic review: impact of health information technology on quality, efficiency, and costs of medical care. Ann Intern Med. 2006;144(10):742-752. https://doi.org/10.7326/0003-4819-144-10-20060516000125

13. Plebani M. Exploring the iceberg of errors in laboratory medicine. Clini Chim Acta. 2009;404(1):16-23. https://doi.org/10.1016/j.cca.2009.03.022

14. Plebani M. Interpretative commenting: a tool for improving the laboratoryclinical interface. Clin Chim Acta. 2009;404(1):46-51. https://doi.org/10.1016/j. cca.2009.03.012

15. Laposata M. Patient-specific narrative interpretations of complex clinical laboratory evaluations: who is competent to provide them? Clin Chem. 2004;50(3):471-472. https://doi.org/10.1373/clinchem.2003.028951

16. Dons RF. Interpretive reporting of laboratory data: proposed criteria to qualify as a high-quality, limited clinical pathology consultation. Endocr Pract. 1996;2(4):255-260. https://doi.org/10.4158/EP.2.4.255

17. Oosterhuis WP, Bruns DE, Watine J, et al. Evidence-based guidelines in laboratory medicine: principles and methods. Clin Chem. 2004;50(5):806-818. https://doi. org/10.1373/clinchem.2003.025528

18. Sacks DB, Bruns DE, Goldstein DE, et al. Guidelines and recommendations for laboratory analysis in the diagnosis and management of diabetes mellitus. Clin Chem. 2002;48(3):436-472. https://doi.org/10.1373/clinchem.2010.161596

19. Elson RB, Connelly DP. Computerized patient records in primary care: their role in mediating guideline-driven physician behavior change. Arch Fam Med. 1995;4(8):698. https://doi.org/10.1001/archfami.4.8.698

20. Smith BJ, McNeely MD. The influence of an expert system for test ordering and interpretation on laboratory investigations. Clin Chem 1999;45(8):1168-1175.

21. Friedman CP, Gatti GG, Franz TM, et al. Do physicians know when their diagnoses are correct? J Gen Intern Med. 2005;20(4):334-339. https://doi.org/10.1111/ j.1525-1497.2005.30145.x

22. Kelly K, Clark B, Brown V, et al. Good practice in the conduct and reporting of survey research. Int J Qual Health Care. 2003;15(3):261-266. https://doi. org/10.1093/intghc/mzg031

23. Kellerman SE, Herold J. Physician response to surveys: a review of the literature. Am J Prev Med. 2001;20(1):61-67. https://doi.org/10.1016/S0749-3797(00)00258-0

24. Berner ES, Graber ML. Overconfidence as a cause of diagnostic error in medicine. Am J Med. 2008;121(5):S2-S3. https://doi.org/10.1016/j.amjmed.2008.01.001 